$\mathrm{Si} \mathrm{Si}$

Nagoya Math. J.

Vol. 114 (1989), 165-172

\title{
A NOTE ON LÉVY'S BROWNIAN MOTION II
}

\author{
SI SI
}

\section{§ 0. Introduction}

The purpose of this paper is to discuss some particular random fields derived from Lévy's Brownian motion to find its characteristic properties of the joint probability distributions. In [9], special attention was paid to the behaviour of the Brownian motion when the parameter runs along a curve in the parameter space, and with this property the conditional expectation has been obtained when the values are known on the curve.

The present paper deals with the variation of the Brownian motion in the normal direction to a given curve, in contrast to the case in [9], where we discussed the properties along the curve. Actually we shall find, in this paper, formulae of the variation with the help of the normal derivative of Brownian motion and observe its singularity. We then discuss partial derivatives of $R^{d}$-parameter Lévy's Brownian motion and make attempt to restrict the parameter to a hypersurface so that we obtain new random fields on that hypersurface. By comparing such derivatives with those of other Gaussian random fields, we can see that the singularity of the new random fields seems to be an interesting characteristic of Lévy's Brownian motion. Further, we hope that our approach may be thought of as a first step to the variational calculus for Gaussian random fields.

\section{§1. Variation of conditional expectation}

We have discussed, in [9], conditional expectation $E[X(P) / C] \equiv$ $E[X(P) / X(A), A \in C]$ of Lévy's Brownian motion $X(P)$ when $X(A), A \in C$, is known, where $P \notin C$ and $C \in C$ :

$$
C=\left\{\text { closed plane curve, } C^{\infty} \text {-manifold }\right\} .
$$

We are now interested in its variation $\delta E[X(P) / C]$ when $C$ varies,

Received February 6, 1988. 
however variational calculus of random functional (of a curve $C$ ) is not well established yet. So we first obtain a representation of $X(A)$ in terms of white noise and use $\mathscr{S}$-transform to have ordinary (non-random) functional of $\xi$, depending on $C$.

Following N.N. Chentsov [11] and H.P. McKean [7], Lévy's Brownian motion can be expressed by

$$
X(A)=c(d) \int_{S(A)}|u|^{-(d-1) / 2} W(u) d u ; \quad u \in R^{d},
$$

in terms of white noise $W$, where $S(A)=\left\{u ;(u, O A) \geq|u|^{2}\right\}$ and

$$
c(d)=\left\{\frac{2(d-1)}{\left|S^{d-1}\right|} \int_{0}^{\pi / 2} \sin ^{d-2} \theta d \theta\right\}^{1 / 2} .
$$

Then the $\mathscr{S}$-transform of $X$ (see [6]) is obtained as

$$
(\mathscr{S} X)(\xi)=c(d) \int \chi_{S(A)}(u)|u|^{-(d-1) / 2} \xi(u) d u .
$$

Let $P$ be fixed. We denote the conditional expectation $E[X(P) / C]$ by $Y(C)$ to emphasize that it is a functional of $C$. It is known (see [9]) that $Y(C)$ can be expressed in the form

$$
Y(C)=\int_{C} f(C, s) X(s) d s,
$$

with sufficiently smooth $f$ for $C \in C$, where $X(s)$ denotes $X(A(s)), A(s) \in C$; $s$ being the parameter taken as an arc length on $C$. The $\mathscr{S}$-transform of $Y(C)$, denoted by $U(C, \xi)$, is obtained as follows.

$$
U(C, \xi)=(\mathscr{S} Y)(\xi)=\int_{C} d s f(C, s) \int g(s, u) \xi(u) d u,
$$

where

$$
g(s, u)=c(d) \chi_{S(A(s))}(u)|u|^{-(d-1) / 2} .
$$

Then the variation of $U(C, \xi)$ exists and has the expression:

$$
\begin{aligned}
\delta U(C, \xi)= & \int d s\{\delta f(C, s)-\kappa f(C, s) \delta n(s)\} \int g(s, u) \xi(u) d u \\
& +\int d s f(C, s) \delta n(s) \int \frac{\partial}{\partial n} g(s, u) \xi(u) d u .
\end{aligned}
$$

Once this formula is established (see [1]), then it guarantees the existence of the stochastic version, namely the existence of $\delta Y(C)$ which is expressed 
in the form

$$
\begin{aligned}
\delta Y(C)= & \int_{C}\{\delta f(C, s)-\kappa f(C, s) \delta n(s)\} X(s) d s \\
& +\int_{C} f(C, s) \frac{\partial}{\partial n} X(s) \delta n(s) d s,
\end{aligned}
$$

where $\frac{\partial}{\partial n}$ stands for the normal differential operator, $\delta n(s)$ denotes the distance between $C$ and $C+\delta C$ at $s, \delta f(C, s)$ denotes the variation of the kernel $f$ and $\kappa$ is the curvature of $C$ (see e.g. [3]).

We do not go into details of $\delta Y(C)$, but having been suggested by the formula (1.7) we investigate, in this paper, the normal derivative $\frac{\partial}{\partial n} X$ and observe its singularity which is to be a characteristic of $X$.

\section{§2. The normal derivative of Lévy's Brownian motion}

Motivated by the variational formula, expressed in the previous section, we introduce the derived random field $\left\{\frac{\partial}{\partial n} X(A), A \in S\right\}$ where $S$ is a hypersurface of $R^{d}$ and where $\frac{\partial}{\partial n} X(A)$ denotes the derivative of Lévy's Brownian motion to the normal direction at a point $A$.

Proposition 1. Let $\{X(\theta) \equiv X(t, \theta), 0 \leq \theta \leq 2 \pi\}$ be Lévy's Brownian motion on a circle $C\left(\subset R^{2}\right)$ with radius $t$. Assume that $C$ passes through the origin. Then the normal derivative $\frac{\partial X}{\partial n}$ on $C$ is neither an ordinary process nor a generalized process, however it is well defined as a generalized process over $R^{2}$.

Proof. The normal derivative of Lévy's Brownian motion at a point $\theta$ on a given circle $C$ is

$$
\frac{\partial}{\partial n} X(t, \theta)=\lim _{\delta t \rightarrow 0} \frac{X(t+\delta t, \theta)-X(t, \theta)}{\delta t} .
$$

Then the covariance of $\frac{\partial X}{\partial n}$ is

$$
\gamma(\alpha)=\frac{\cos ^{2} \alpha / 2}{4 t \sin |\alpha / 2|}
$$

which is not well defined when $\alpha$ tends to zero. So we can see that 
$\frac{\partial X}{\partial n}$ is neither an ordinary process nor a generalized process. Let

$$
N(\xi)=\iint_{R^{2}} \frac{\partial}{\partial r} X(r, \theta) \xi(r, \theta) r d r d \theta,
$$

with $\xi \in \mathscr{S}\left(R^{2}\right)$. Then we can easily prove that $N(\xi)$ is a stationary Gaussian process. Thus the assertion follows.

It can easily be seen that the same situation occurs for $C \subset R^{2}, C$ being (i) a straight line (or) (ii) a general curve in $C$. It means that the covariance function of normal derivative of Lévy's Brownian motion on $C$ has the same singularity of order -1 in the cases (i) and (ii) above.

Theorem. Let $X(A), A \in R^{d}$, be a Gaussian random field such that

$$
E[X(A)]=0, \quad E\left[(X(A)-X(B))^{2}\right]=c \rho(A, B)+\varphi(\rho(A, B)), \quad c>0,
$$

where $\rho$ is the Euclidean distance in $R^{d}$ and where $\varphi$ is a $C^{2}(0, \infty)$-function with $\varphi(0)=\varphi^{\prime}(0)=0$. Let $S$ be $a(d-1)$-dimensional surface in $R^{d}$ which is an analytic manifold (including the case of a $(d-1)$-dimensional hyperplane). Then

(i) for $d=2,\left.\frac{\partial X}{\partial n}\right|_{S}$ is not well defined, however $\frac{\partial X}{\partial n}$ is defined as a generalized Gaussian random field over $R^{2}$,

(ii) for $d>2,\left.\frac{\partial^{k} X}{\partial n^{k}}\right|_{s}, k \leq \frac{d-1}{2}$, exist as generalized Gaussian random fields over $S$,

where $\frac{\partial}{\partial n}$ denotes the normal derivative and $\left.\frac{\partial X}{\partial n}\right|_{s}$ denotes the restriction of $\frac{\partial X}{\partial n}$ to the manifold $S$.

Proof. We can see from actual computation that the covariance function of $\frac{\partial X}{\partial n}$ has singularity of order -1 . Thus for $d=2$, the situation is the same as the above proposition and so the assertion (i) is obvious. For higher dimensional parameter space (i.e. $d>2$ ), it is clear that

$$
N(\xi)=\int_{R^{d}} \frac{\partial}{\partial n} X\left(u_{1}, \cdots, u_{d}\right) \xi\left(u_{1}, \cdots, u_{d}\right) d u ; \quad \xi \in \mathscr{S}\left(R^{d}\right)
$$


is well defined as an $R^{d}$-parameter generalized Gaussian random field. Even we can restrict $N(\xi)$ to a $(d-1)$-dimensional manifold $S$, described in the theorem statement. Thus $\left.\frac{\partial X}{\partial n}\right|_{S}$ is defined as a generalized Gaussian random field. We can easily prove that $\frac{\partial^{k} X}{\partial n^{k}}$ is also a generalized Gaussian random field for $k \leq \frac{d-1}{2}$.

Here we should note that Lévy's Brownian motion is the most important example for which (2.3) holds. Another interesting example is the one for which (2.3) holds with

$$
\varphi(t)=\int_{0}^{\infty}\left(1-e^{-\lambda t^{2}}\right) d \sigma(\lambda), \quad \int_{0}^{\infty} \lambda d \sigma(\lambda)<\infty .
$$

\section{§3. Comparison with Wiener and Ornstein Uhlenbeck processes}

We obtain partial derivatives of Wiener and Ornstein Uhlenbeck processes and observe their singularities to compare with the Lévy Brownian motion case, where the covariance function has singularity of order -1 . We see that normal derivative over a hypersurface is one of the significant characteristics of Gaussian random fields, although far from the complete characterization.

\section{1) Wiener process}

Let $\boldsymbol{Y}=\left\{Y(u) ; u=\left(u_{1}, \cdots, u_{d}\right) \in\left(R_{+}\right)^{d}\right\}, d \geq 2$, be Wiener process. Namely, $\boldsymbol{Y}$ is a Gaussian random field such that

$$
E[Y(u)]=0, \quad E[Y(u) Y(v)]=\prod_{i=1}^{d}\left(u_{i} \wedge v_{i}\right) ;
$$

where $u=\left(u_{1}, \cdots, u_{d}\right)$ and $v=\left(v_{1}, \cdots, v_{d}\right)$.

Proposition 2. (i) Let $S$ be $\left\{u=\left(u_{1}, \cdots, u_{i}, \cdots, u_{d}\right) \in\left(R_{+}\right)^{d} ; u_{i}=c\right\}$, for $1 \leq i \leq d$. Then the derived process restricted to $S,\left.\left(\frac{\partial}{\partial u_{k}} Y(u)\right)\right|_{S}$ is

(a) not well defined if $k=i$,

(b) well defined as a generalized Gaussian random field if $k \neq i$.

(ii) The normal derivative of Wiener process $\left.\left(\frac{\partial}{\partial n} Y(u)\right)\right|_{S}$, where $S$ is a spherical surface $S^{d-1}$ which is a subset of $\left(R_{+}\right)^{d}$, is well defined as a generalized Gaussian random field. 
Here it is noted that, both Lévy's Brownian motion and Wiener process give us a Brownian motion (up to constant factor) with one dimensional paramter if the parameter of these processes is restricted to a straight line. And the derivatives of these fields along a straight line are white noises. However, if the partial derivatives are taken to be the normal direction to some $(d-1)$-dimensional manifolds we can see that Lévy's Brownian motion and Wiener process present entirely different features.

\section{2) Ornstein Uhlenbeck process}

Let $\left\{U_{m}(u) ; u=\left(u_{1}, \cdots, u_{d}\right) \in R^{d}\right\}, d \geq 2$, be Ornstein Uhlenbeck process with mass parameter $m>0$. We know that it is a generalized Gaussian random field with the characteristic functional

$$
C(\xi)=\exp \left[-\frac{1}{2} \int_{R^{d}} \frac{|\hat{\xi}(\lambda)|^{2}}{m^{2}+|\lambda|^{2}} d \lambda\right]
$$

in which $\hat{\xi}$ is the Fourier transform of $\xi$.

Proposition 3. The normal derivative $\frac{\partial U_{m}}{\partial n}$ is again a generalized Gaussian random field, but the restriction of which to a hyperplane is not well defined.

Observation. The normal derivative $\left.\frac{\partial U_{m}}{\partial n}\right|_{R^{d-1}}$ can be viewed as a superposition of mutually independent $(d-1)$-dimensional known random fields $U_{\alpha(m, \lambda)}$, with $\alpha(m, \lambda)=\sqrt{\left(m^{2}+\lambda^{2}\right)}$, such as a limit of

$$
\int_{0}^{N} \lambda U_{\alpha(m, \lambda)} d \lambda
$$

as $N \rightarrow \infty$.

Remarks. (i) The restriction of the normal derivative of $R^{2}$-parameter Ornstein Uhlenbeck process to a circle $S$ is not well defined as in the case of Lévy's Brownian motion, however the awkwardness appears in a quite different manner.

(ii) Unlike Lévy's Brownian motion the singularity always occurs in $R^{d}$-parameter case for every $d \geq 2$. 


\section{§4. Discussion}

As is noted in Introduction, we have discussed movement of Lévy's Brownian motion when the parameter varies in the normal direction to the specified manifold. Now, in this line, we would like to show one more class of random fields which has been introduced in [5] and admits us to take its variation. Namely, it is the class involving such a process as

$$
X(C)=\int_{[C]} g(u) W(u) d u=\int \chi_{[C]}(u) g(u) W(u) d u,
$$

where $[C]\left(\subset R^{2}\right)$ is the domain enclosed by a curve $C$ in $C, \chi$ denotes the indicator function, $g$ is an $L^{2}$-function, and where $W(u)$ denotes the two parameter white noise. Then its variation, when a curve $C$ changes to $C+\delta C(\in C)$, is obtained as

$$
\delta X(C)=\int_{C} g(s) \delta n(s) W(s) d s,
$$

where $W(s)$ and $g(s)$, taking $s$ to be the arc length on $C$, are white noise and the value of $g$ at the point parametrized by $s$, respectively, and where $\delta n(s)$ is the distance between $C$ and $C+\delta C$ (for detail see [5]). In fact, the formula (4.2) shows that $\delta X(C)$ plays a role of, so-to-speak, an innovation of $X(C)$, since the white noise integral extends only over $C$. In view of this property, $X(C)$, given by the formula (4.1), may be called an additive Gaussian process depending on the parameter $C$.

What we have discussed so far on this topic can easily be generalized to the class of additive Gaussian processes depending on $C \in C$, which is a class of $(d-1)$-dimensional $C^{\infty}$-manifolds in $R^{d}$ without boundary.

Acknowledgement. The author wishes to express her gratitude to Professor T. Hida and Professor A. Noda for their valuable suggestions and comments.

\section{REFERENCES}

[1] P. Lévy, Problèmes concrets d'analyse fonctionelle, Gautheir-Villars, 1951.

[2] T. Hida, Lévy's functional analysis and stochastic analysis, Lecture notes, Nagoya University, 1986; notes by Si Si.

[ 3 ] —-, Lévy's functional analysis and stochastic calculus, preprint, (1987).

[4] T. Hida and L. Streit, On quantum theory in terms of white noise, Nagoya Math. J., 68 (1977), 21-34. 
[ 5 ] T. Hida, K.-S. Lee and Si Si, Multi-dimensional parameter white noise and Gaussian random fields, Recent advances in communications and control theory, 1987, ed. R. E. Kalman, G. I. Marchuk, A. E. Ruberti and A. J. Viterbi, 177-183.

[6 ] I. Kubo and S. Takenaka, Calculus on Gaussian white noise I-IV, Proc. Japan Acad., 56A (1980), 376-380, 411-416, 57A (1981), 433-437, 58A (1982), 186-189.

[ 7 ] H. P. McKean, Jr., Brownian motion with a several dimensional time, Theory Probab. Appl., 8 (1963), 335-354.

[ 8 ] A. Noda, Generalized Radon Transform and Lévy's Brownian motion I, II, Nagoya Math. J., 105 (1987), 71-87, 89-107.

[ 9 ] Si Si, A note on Lévy's Brownian motion, Nagoya Math. J., 108 (1987), 121-130.

[10] - Gaussian processes and conditional expectations, BiBoS notes, Nr. 292/87, Universität Bielefeld, 1987.

[11] N. N. Chentsov, Lévy Brownian motion for several parameters and generalized white noise, Theory Probab. Appl., 2 (1957), 265-266 (English translation).

[12] A. Goldman, Techniques biharmoniques pour l'etude du mouvement brownien de P. Lévy a trois parametres, preprint.

Department of Mathematics

Faculty of Science

Nagoya University

Nagoya 464, Japan

Current address:

Department of Mathematics

Rangoon University

Rangoon, Burma 